\title{
Carbon Nitride Thin Films as All-In-One Technology for Photocatalysis
}

\author{
Stefano Mazzanti, Giovanni Manfredi, Alex J. Barker, Markus Antonietti, Aleksandr Savateev,* \\ and Paolo Giusto*
}

Cite This: ACS Catal. 2021, 11, 11109-11116

Read Online

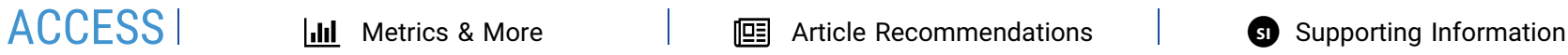

ABSTRACT: Organic $\pi$-conjugated polymers are promising heterogeneous photocatalysts that involve photoredox or energy transfer processes. In such settings, the materials are usually applied in the form of dispersion in liquid medium, which is bound to certain technological limits of applicability. Herein, we present an innovative approach using carbon nitride thin films prepared via chemical vapor deposition at different vessel walls and using them as batch and microfluidic photoreactors. This approach allows us not only to fabricate technologically relevant and reusable devices but also to improve

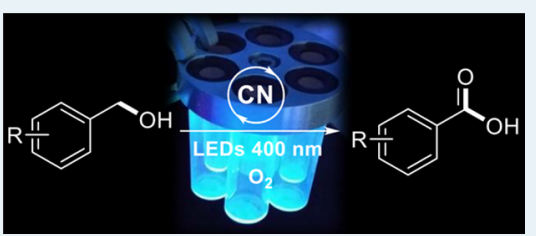
photophysical properties of carbon nitride, such as the singlet-triplet energy gap and lifetime of triplet excited states, when the material is assembled in thin films. These morphological changes are employed to maximize the performance of the materials in photocatalytic reactions, in which the carbon nitride thin films show at least 1 order of magnitude higher activity per area unit compared to photocatalysis using suspended particles.

KEYWORDS: carbon nitride, chemical vapor deposition, flow chemistry, photocatalysis, thin films

\section{INTRODUCTION}

Photoredox catalysis enables unique reaction pathways to high value-added products that are not available with classical thermal activation of the molecules. ${ }^{1-4}$ Recently, visible light photocatalysis has gained high momentum, not only in academic research but also in the chemical industry as a new synthetic tool, ${ }^{5,6}$ in the preparation of fine chemicals and active pharmaceutical ingredients. ${ }^{7-10}$

In general, photoredox catalysis can be divided into homogeneous and heterogeneous catalyses, both effectively mediating a number of photochemical transformations. ${ }^{11}$ However, each of these approaches possesses unique advantages and disadvantages. For example, homogeneous photocatalysis provides very high density of redox sites per volume unit in solution. On the other hand, a unique feature of heterogeneous photocatalysis is tuning properties of the employed material, which typically is a semiconductor, by adjusting its morphology. In other words, semiconductor particles with essentially the same bulk chemical composition, but having, for example, variable diameter, ${ }^{12}$ aspect ratio between different facets of the single crystal, ${ }^{13}$ just to name a few, ${ }^{14}$ possess different photophysical properties and therefore demonstrate different activities.

Despite an outcome of a certain photocatalytic reaction mediated by either the homogeneous or heterogeneous photocatalyst is the same, the underpinning mechanism is quite often different. ${ }^{15}$ In homogeneous organic photoredox catalysis, represented, for example, by organic dyes, triplet excited states of the sensitizer with lifetime in the $\mu \mathrm{s}-\mathrm{ms}$ range are often engaged. ${ }^{16}$ While inorganic semiconductors do not possess triplet states, organic $\pi$-conjugated systems fall in between inorganic semiconductors, in terms of their heterogeneous nature and processability, and molecular sensitizers, due to similarities of their local structure. Carbon nitride $(\mathrm{CN})$, a polymeric semiconductor with the ideal formula $\mathrm{C}_{3} \mathrm{~N}_{4}$, constituted by heptazine or triazine units crosslinked via nitrogen bridges and stacked in a graphitic fashion, is one type of such materials. ${ }^{17,18}$ It has been actively studied in organic photocatalysis. ${ }^{19,20}$ For example, it has been shown that upon excitation, carbon nitrides undergo singlet-triplet intersystem crossing (ISC) and form triplet excited states with the lifetime of up to hundreds of microseconds. ${ }^{21-23}$ In organic photocatalysis, triplet states in carbon nitrides have been employed in energy transfer (EnT) to $\mathrm{O}_{2}$ in the synthesis of oxadiazoles-1,2, $4^{21}$ and sensitization of the $\mathrm{Ni}$ (II) co-catalyst in arylation of $\mathrm{C}\left(\mathrm{sp}^{3}\right)-\mathrm{H}$ bonds in amides. ${ }^{24}$

Semiconductor photocatalysis also offers multiple engineering solutions. ${ }^{25}$ For example, coating of the photoreactor wall with semiconductor particles is an appealing feature in combination with flow technology. ${ }^{26}$ In this regard, Wang et al. recently reported on a fixed-bed flow system employing $\mathrm{CN}$ supported on glass fibers and beads. ${ }^{27}$ However, backscattering

Received: June 29, 2021

Revised: August 6, 2021

Published: August 20, 2021 
from the surface limits accessibility of photons and reduces efficiency of the reactor.

For high-throughput screening (HTS) in photocatalysis and flow technology, an ideal solution would be a thin transparent layer of the semiconductor deposited at the inner wall of the photoreactor. Thermal chemical vapor deposition (CVD) offers an elegant solution to grow such thin films by reacting precursors in the gas phase followed by deposition at the surface of the desired substrate. ${ }^{28}$ Indeed, the approach has been validated in the preparation of $\mathrm{CN}$ thin films. ${ }^{29,30}$ Carbon nitride thin films deposited at conductive substrates by CVD are applied mainly as photoelectrodes demonstrating current densities of several hundreds $\mu \mathrm{A} \mathrm{cm} \mathrm{cm}^{-2}$, IPCE $>12 \%$, and faradaic efficiency toward $\mathrm{O}_{2}$ of $40.3-51 \%$ at $+1.23 \mathrm{~V}$, which underlines robustness, reproducibility, and operational simplicity of the method. ${ }^{31-34}$

Very recently, our group reported a method to obtain highly homogeneous $\mathrm{CN}$ thin films over areas of several dozens of $\mathrm{cm}^{2}$ on different substrates, regardless of their shape and surface topology by means of CVD under highly controlled conditions of vacuum, gas flow, and temperature. ${ }^{35,36}$ High homogeneity and flatness allowed for applying these $\mathrm{CN}$ thin films in optics, showing that they have a very high refractive index comparable to that of diamond in the visible range.

In the last decade, $\mathrm{CN}$ as a material in general has been studied in water splitting, production of hydrogen, ${ }^{37}$ conversion of $\mathrm{CO}_{2}$ into valuable products, ${ }^{38}$ and synthesis of fine chemicals. ${ }^{39-44}$ Due to the exciting properties of $\mathrm{CN}$, the scope of applications for this material spans beyond photocatalysis, for example, to optoelectronics, ${ }^{45,46}$ energy and membrane separation, ${ }^{47}$ among others. ${ }^{48,49}$

Rich experimental data of using $\mathrm{CN}$ particulates in photocatalysis, as well as unique opportunities offered by this class of materials in combination with the CVD, enabled us to explore the highly homogeneous thin and transparent $\mathrm{CN}$ film as active coating in photoreactors.

Herein, we present an innovative approach in photocatalysis using nanometer-thick $\mathrm{CN}$ films deposited on different glass surfaces, such as standard glass vials and the microfluidic reactor plate, by means of CVD. The glassware with the photocatalytically active layer is tested in the oxidation of benzyl alcohols under light irradiation.

\section{RESULTS AND DISCUSSION}

We coated standard $4 \mathrm{~mL}$ glass vials that are also used by many research groups working in the field of automated photoredox catalysis across the world, with the $\mathrm{CN}$ thin film using CVD similar to the earlier reported procedure. ${ }^{35,36}$ In this way, visible batch wall reactors (Vis-BWR) were fabricated (Figure 1a). Deposition of the $\mathrm{CN}$ film on the reactor wall after the CVD is seen with naked eye by its typical yellowish color, photonic reflections, and the deep blue fluorescence when the Vis-BWR is exposed to UV light (Figure 1b). ${ }^{50}$ The CN film strongly absorbs photons with wavelength $\leq 420 \mathrm{~nm}$ as confirmed by the UV-vis absorption spectrum (Figure S1a). In the steady-state photoluminescence spectrum, the maximum is observed at $460 \mathrm{~nm}$ (Figure S1b).

A CN film with a thickness of about $111 \mathrm{~nm}$ was obtained as evidenced by atomic force microscopy (AFM) (Figure S2). $\mathrm{CN}$ coating in the Vis-BWR is homogeneous and flat, while surface roughness is $<1 \mathrm{~nm}$ over the field of view $5 \times 5 \mu \mathrm{m}^{2}$, as confirmed by AFM (Figure S3) and scanning electron microscopy (SEM, Figure 2a). Despite the fluorescence

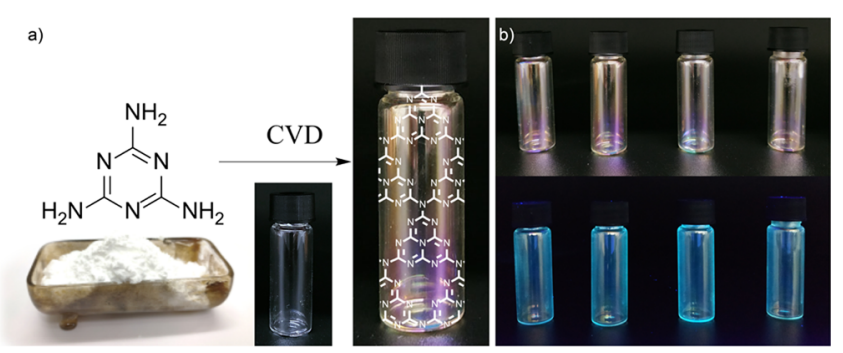

Figure 1. Fabrication of Vis-BWR and their appearance. (a) Schematic representation of the CVD process of vial coating with $\mathrm{CN}$, showing the ideal heptazinic structure. (b) Vis-BWR under visible and $365 \mathrm{~nm}$ light.

quantum yield of the $\mathrm{CN}$ films is relatively low $(<0.6 \%)$, emission can be observed even by naked eye. For further analysis, the $\mathrm{CN}$ film was completely removed from the selected area of the vial by mechanical treatment. By comparing the cleaned area of the vial with the untreated area after the CVD, the presence of the $\mathrm{CN}$ film on the glass surface was confirmed by energy-dispersive $\mathrm{X}$-ray spectroscopy (EDX) mapping (Figure $2 \mathrm{~b}-\mathrm{d}$ ). ${ }^{51}$ At the nanoscale, the film reveals the presence of a layered structure, where each shade of gray represent a stack of layers in graphitic-like fashion (Figure 2e). The graphitic-like structure was further confirmed by Xray diffraction, where we identified the $\pi-\pi$ stacking peak at $27.4^{\circ}$ (Figure S5). Based on the interlayer distance of $0.33 \mathrm{~nm}$ and film thickness of $111 \mathrm{~nm}$, we estimate the average number of layers in the $\mathrm{CN}$ film to be ca. 350. Electron energy loss spectroscopy (EELS) reveals the presence of carbon (as a peak at $287 \mathrm{eV})$ and nitrogen $(400 \mathrm{eV})$, which are integrated in the strongly $\mathrm{sp}^{2}$-conjugated structure as confirmed by the pronounced $\pi^{*}$ peak (Figure 2f). The surface of the CN film is free of oxygen contaminations as concluded from the absence of a peak at ca. $532 \mathrm{eV}$ (Figure 2f), which is in good agreement with previous results. ${ }^{35,52}$ In the present study, EELS provides a qualitative analysis on the type of bonds and conjugation. The measurement is not quantitative because in anisotropic systems, the response depends not only on the magnitude of the applied energy but also on the orientation of the sample. ${ }^{53,54}$

The high quality of the deposition achieved on the vials provides a solid evidence to transform, in principle, every piece of lab glassware, such as vials, test tubes, and flasks, into a VisBWR, according to the reaction requirements.

CVD allows growing $\mathrm{CN}$ thin films, while at the same time, the technique does not alter substantially the positions of the highest occupied molecular orbital and lowest unoccupied molecular orbital, ${ }^{35}$ which in combination with numerous earlier reports using the suspended $\mathrm{CN}$ particulate, enables us to consider such films as very promising candidates for using them as photocatalysts in net oxidative reactions under visible light. Taking into account the innovative approach used in this work, we have chosen oxidation of benzyl alcohols to benzoic acid as a benchmark reaction to compare the performance of the $\mathrm{CN}$ thin films and Vis-BWRs with other photocatalysts and photocatalytic systems. Acetonitrile was chosen as a solvent due to its polarity and high capacity toward dissolution of oxygen and high chemical stability against reactive oxygen species, such as superoxide radicals and singlet oxygen. ${ }^{55}$ Thorough optimization of reaction conditions is given in the Supporting Information (Table S1). Control experiments confirmed that the reaction is purely photocatalytic. Thus, 

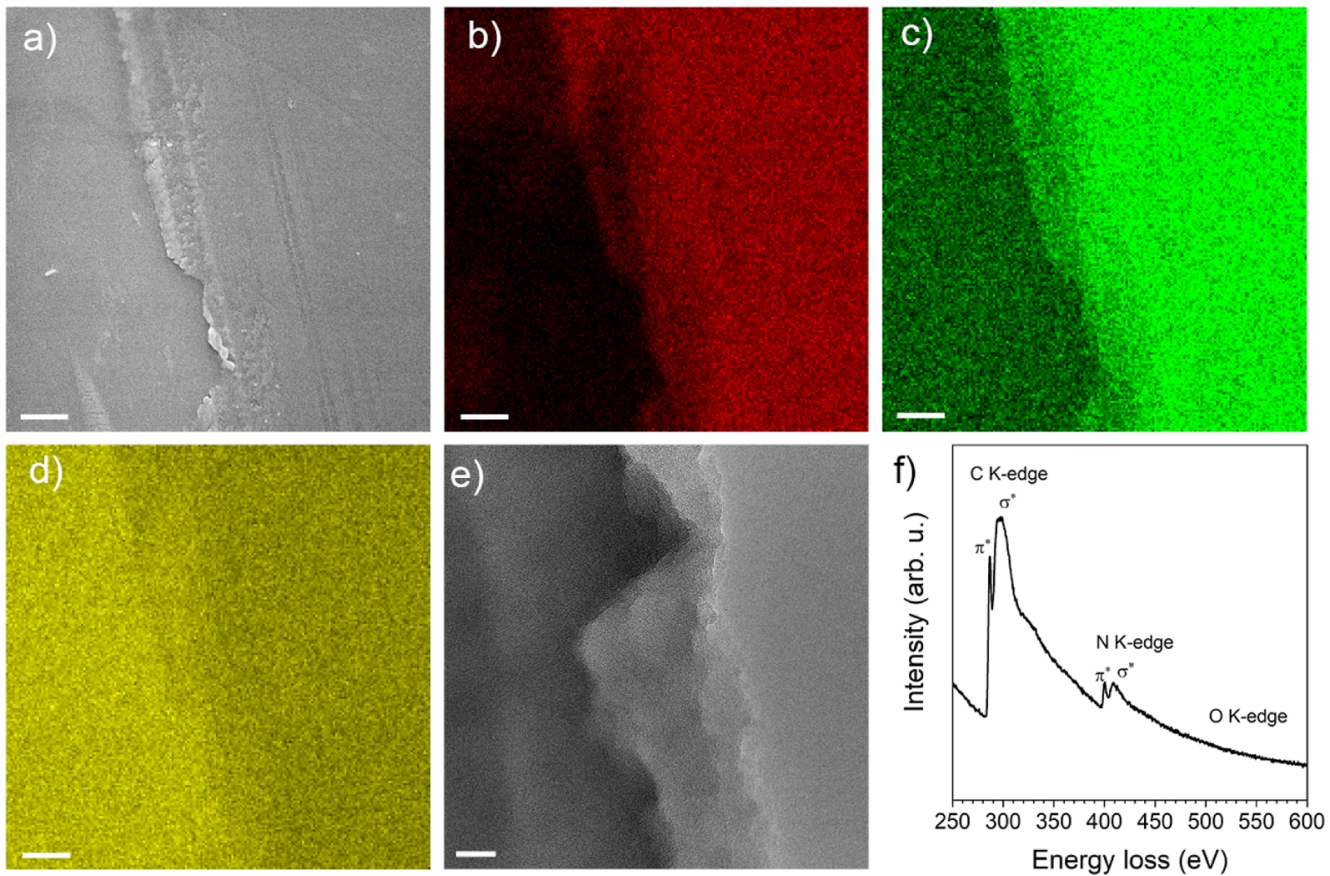

Figure 2. CN film characterization. (a) SEM image of a scratched area of the coated vial. (b-d) SEM-EDX mapping of carbon (in red), nitrogen (in green), and silicon (in yellow); scale bar $2 \mu \mathrm{m}$. (e) TEM image of the CN thin film (scale bar $20 \mathrm{~nm}$ ) and (f) EELS core loss spectrum.

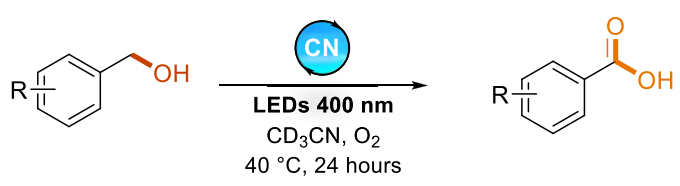

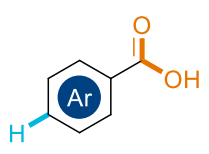

3a, $87 \%(100 \%)$

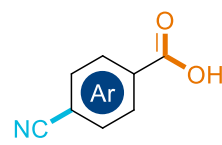

3 e $62 \%(100 \%)$

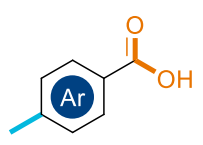

3b, $69 \%(99 \%)$

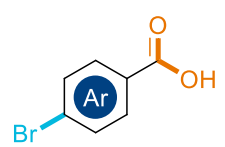

$3 c, 66 \%(91 \%)$

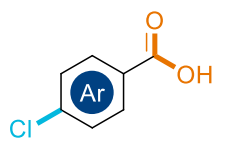

3d, $76 \%(85 \%)$

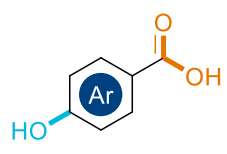

3i 3\% (100\%)

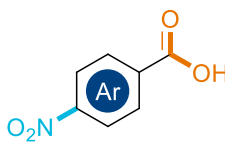

3f $76 \%(100 \%)$<smiles>O=C(O)c1ccc(C(F)(F)F)cc1</smiles>

3g $85 \%(100 \%)$

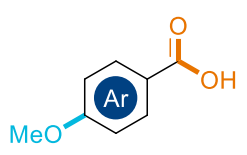

3h $33 \%(100 \%)$

Figure 3. Scope of benzyl alcohols. Reaction conditions: alcohol ( $0.01 \mathrm{mmol}), \mathrm{CD}_{3} \mathrm{CN}(3 \mathrm{~mL}), \mathrm{O}_{2}(1 \mathrm{bar})$, reaction was conducted in $4 \mathrm{~mL}$ VisBWR. See the experimental procedure for details in the Supporting Information. Yields and conversions (in parentheses) have been determined using 1,3,5-trimethoxybenzene as an internal standard by ${ }^{1} \mathrm{H}$ NMR.

no benzoic acid was obtained in the absence of the $\mathrm{CN}$ films, excluding the thermal effects induced by heating originating from the light source as a potential driving force (entry 14). Reaction did not proceed in the dark either (entry 15). Under optimized conditions, benzoic acid was obtained in $87 \%$ yield along with the quantitative conversion of benzyl alcohol under illumination with $400 \mathrm{~nm}$ LED for $24 \mathrm{~h}$. Recently, Hutchings et al. showed that benzyl alcohol acts as a quencher of the radical species in the thermal oxidation of benzaldehyde to benzoic acid, even at very low concentrations, which explains 
the formation of benzaldehyde in our case at lower conversions (Table S1, entries 1-12, Figure S7). ${ }^{56}$

After finding optimal reaction conditions, we expanded the scope of benzyl alcohols (Figure 3). The method provides a simple procedure for the preparation of substituted benzoic acids. Substrates bearing poorly electron donating or electron withdrawing groups at the aromatic ring have been converted into the corresponding benzoic acids in good and high yields $(3 \mathbf{a}-\mathbf{g})$. On the other hand, alcohols bearing strong electron donating groups were fully consumed, but the corresponding acids were obtained in poor or no yields $(3 \mathbf{h}-\mathbf{k})$. Lower tolerance of the functional groups to reactive oxygen species is apparently responsible for lower selectivity in these cases (Figure S8 and S9). ${ }^{57}$

The conventional CVD method allows for fabricating several vials at once with high reproducibility, which makes the technology highly relevant to the needs of high-throughput photocatalysis (Figure 4a). ${ }^{58}$ In addition, Vis-BWR coated with the $\mathrm{CN}$ thin film are stable and can be reused many times, here proven only for three to five catalytic cycles (Figure $4 b$ ).

To evaluate the performance of the $\mathrm{CN}$ films and compare it with that of other photoredox catalysts, we determined the apparent quantum yield (AQY). Vis-BWR showed an AQY of $1.8 \%$, which is higher compared to that of commonly used Ir and $\mathrm{Ru}$ sensitizers (Figure S11). The AQY of Vis-BWR is comparable to that of mesoporous graphitic-CN (mpg-CN)
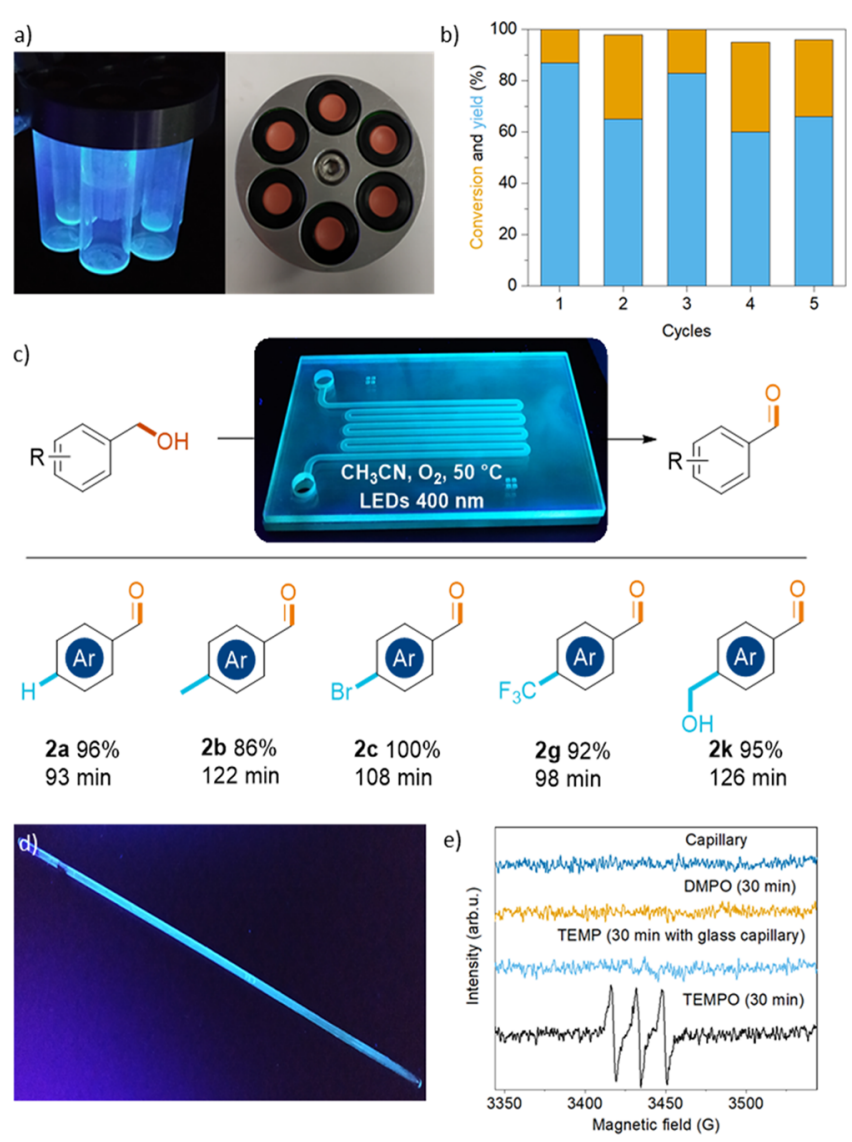

Figure 4. Application of the $\mathrm{CN}$ thin film in photocatalysis. (a) VisBWR for high-throughput screening. (b) Recyclability of Vis-BWR. (c) Scope of benzyl alcohols in flow (inset shows the half cell of VisFWR coated with the CN film under $365 \mathrm{~nm}$ light, see Supporting Information for more details). (d) Coated capillary. (e) TEMPO generation and detection via in situ EPR. and it is higher than for potassium poly(heptazine)imide ( $\mathrm{K}$ PHI). ${ }^{39,59}$ Additionally, Vis-BWR shows higher activity per area unit compared to dispersions of semiconductor particles (see Figure S12 and the relevant discussion therein). Explanation of enhancement of the activity is given below taking into account the results of time-resolved spectroscopic characterization of the $\mathrm{CN}$ thin films.

Vis-BWRs having different thicknesses of the CN film (123, 111 , and $55 \mathrm{~nm}$ ) were also prepared to investigate the influence of this parameter on the photocatalytic activity (Figure S13). The data show that the thicker the CN film, the faster is kinetics, which in turn leads to higher selectivity toward benzaldehyde (Figure S14a). This study agrees with the fact that the number of available excitons correlates with the number of absorbed photons, and thus, it is proportional to the number of photoactive layers. Although the geometric surface area of the $\mathrm{CN}$ films is the same, the efficiency is improved by increasing the number of layers (thickness). The steady-state fluorescence spectra of Vis-BWRs show that there are fewer recombination events in thinner films, which probably stems from the fact that excitons are more likely to reach the film surface (Figure S14b). Nevertheless, faster kinetics of benzyl alcohol oxidation in case of thicker films indicates that recombination of excitons as a side process does not contribute significantly to the overall efficiency.

We also investigated the possible chemical modifications of the carbon nitride surface of the BWR during the reaction. The steady-state UV-vis absorption spectrum of the BWR after five photocatalytic cycles showed a shift of the absorption edge to shorter wavelengths, but no additional features in the visible range evolved (Figure S15a). A slight shift of the emission maximum in the PL spectrum was observed in the case of the BWR (Figure S15b). Such observations are explained bythe partial exfoliation of the thin films from the vessel walls after five catalytic experiments rather than chemical modification of the carbon nitride film. This conclusion is also in agreement with the investigation of a series of films of variable thickness, where photoluminescence and catalytic activity decrease by decreasing the number of photoactive layers (Figure S14).

Immobilization of the $\mathrm{CN}$ films at the glass surface simplifies the work-up procedure and more importantly eliminates the necessity of photocatalyst separation. These features prompted us to study the CN films in combination with flow technology. Microfluidic reactors are a promising class of chemical reactors, which offer significant advantages as compared to batch reactors, such as larger scale production (seamless scalability), precise control over the residence time, and local stoichiometry. ${ }^{60}$ Here, two mirror parts of a microfluidic reactor (channel diameter $250 \mu \mathrm{m}$ ) were coated homogeneously with the $\mathrm{CN}$ thin films on the channel walls to obtain a visible (microfluidic) flow wall reactor (Vis-FWR) (Figure 4c). The Vis-FWR was again tested with the oxidation of benzyl alcohols 1a-c, 1g, and 1k under illumination with $400 \mathrm{~nm}$. In this setting, the main products are benzaldehydes $\mathbf{2 a}-\mathbf{c}, \mathbf{2 g}$, and $2 \mathbf{k}$ in excellent yields $86-100 \%$ with much shorter reaction time, applying recirculation of the reaction mixture (Figure 4c). In this configuration, the reaction proceed at higher conversion rates due to the higher surface area/volume ratio in the microchannels, preventing the consecutive oxidation of aldehyde to the corresponding acid. Oxidation of $\mathbf{1 a}$ in flow to benzaldehyde $\mathbf{2 a}$ follows quasi zero order kinetics, probably due to the photon limited regime (Table S3, Figure S16). 
To gain insights into the nature of reactive oxygen species in the photocatalytic oxidation of benzyl alcohol and to propose a mechanism, we applied in situ EPR spectroscopy using a glass capillary coated with the CN film by CVD, in the same way as the Vis-BWR and Vis-BFR were produced (Figure 4d). The capillary was charged with the solution of 2,2,6,6-tetramethylpiperidine in the $\mathrm{O}_{2}$ saturated solution of $\mathrm{MeCN}$ followed by irradiation with $415 \mathrm{~nm}$ in the cavity of the EPR spectrometer. Appearance of the TEMPO signal indicates that in this set of experiments, $\mathrm{CN}$ films sensitize singlet oxygen $\left({ }^{1} \mathrm{O}_{2}\right)$ (Figure $4 \mathrm{e}$, see Supporting Information for further details).

Eventually, we applied time-resolved spectroscopy to gain insights into the changes of $\mathrm{CN}$ excited-state dynamics, which result from the assembly of this material into thin films. Thus, photophysics of the excited state was investigated using timecorrelated single photon counting time-resolved photoluminescence (tr-PL) and transient absorption spectroscopy (TAS). PL decay is well fitted $\left(R^{2}>0.99\right)$ using a single stretched exponential function with a time exponent of half and a lifetime of $\sim 0.6$ ns (Figure S17, Table S4). Such behavior is common for polymer systems that present defects and traps (primary and secondary amines as terminal groups in this case), ${ }^{61,62}$ in good agreement with the recorded relatively low internal quantum efficiency of $0.6 \%$ (Figure S1b). ${ }^{63}$

According to the TAS data acquired under $\mathrm{N}_{2}$ (Figure S18), excitation of the $\mathrm{CN}$ film leads to the appearance of a broad band in the observed wavelength range (500-750 nm) ascribed to excited state absorption (ESA). ${ }^{64}$ Such behavior of the $\mathrm{CN}$ thin films is significantly different from the dispersion of $\mathrm{CN}$ in the liquid phase reported earlier. ${ }^{21,61}$ Therein, a negative photoinduced $\triangle \mathrm{OD}$ signal in the visible range was attributed to ground-state bleaching (GSB) of the $\mathrm{CN}$, whereas we observe a positive $\triangle \mathrm{OD}$ signal from the $\mathrm{CN}$ thin films, indicating a transient photoinduced absorption. ${ }^{61}$ Therefore, in case of the $\mathrm{CN}$ film, the net signal composed of the CN GSB and ESA is positive.

Analysis of the ESA decay in the femto- to microsecond time scale suggests that the process is characterized by two lifetimes (Figure S19). The faster process is described by the same parameters $(\beta=1 / 2$ and $\tau=0.6 \mathrm{~ns})$ as tr-PL, which suggest that the singlet excited states $\left(S_{1}\right)$ of the $\mathrm{CN}$ possess broad absorption in the visible range (Table S5). We assume that the depopulation of $S_{1}$ in the $\mathrm{CN}$ film proceeds via three pathways: (i) radiative relaxation, (ii) singlet-triplet ISC, and (iii) nonradiative recombination. Taking into account extremely low fluorescence quantum yields, $<0.6 \%$ (Figure S1b), the first pathway is not dominant. The second, slower process, of the $\mathrm{CN}$ ground-state recovery corresponds to the depopulation of the triplet excited states $\left(\mathrm{T}_{1}\right)$ in the $\mathrm{CN}$ film. Furthermore, a channel by which $T_{1}$ is converted back into $S_{1}$, such as triplettriplet annihilation (TTA, which would exhibit a densitydependent rate), explains the intensity dependence of the TA recombination rate in the nano- to microsecond time scale (Figure S20) and the longer time constants obtained, when the CN film was in degassed acetonitrile (Table S6). ${ }^{22}$ TTA also contributes to the tail of long-lived emission seen in tr-PL, although the significant stretching rates of both PL and TA kinetics is likely due more to disorder in the excitonic landscape of CN materials. ${ }^{65}$ The absence of the noticeable peak shift in the TAS indicates a vanishingly small singlettriplet energy gap, which might be the additional pathway of $\mathrm{S}_{1}-\mathrm{T}_{1}$ interconversion.
Overall, different photophysics of the $\mathrm{CN}$ thin films, compared to the reported earlier, obviously stems from different synthesis conditions-polycondensation of the precursor in bulk at atmospheric pressure (used earlier) ${ }^{61}$ and polycondensation-deposition of carbon nitride in thin layers from vacuum under the controlled conditions in the CVD system (gas-to-solid reaction in this work). Apart from being indispensable to create durable technological devices, $\mathrm{CVD}$ also produces the $\mathrm{CN}$ material with narrow $\Delta E_{\mathrm{ST}}$, which facilitates singlet-triplet ISC. Until now, $\mathrm{CN}$ materials prepared by condensation of the precursors in bulk have been reported to have a $\Delta E_{\mathrm{ST}}$ value of $0.243-0.248 \mathrm{eV}^{21,22}$ Furthermore, the absence of voids in the structure of the $\mathrm{CN}$ thin film is also beneficial to suppress the nonradiative recombination of triplet excited states (vibrational relaxation) by decreasing free volume in the crystal lattice and surface defects. ${ }^{66,67}$ These differences strongly point that a strict control over preparation conditions of the photocatalysts defines the nanostructure of the sensitizer and influences the material photophysical properties to achieve high activity in photocatalytic reactions that involve EnT.

We combine the results of the spectroscopic study with experimental data and propose the following mechanism of benzyl alcohol oxidation by the $\mathrm{CN}$ thin films (Figure 5a).

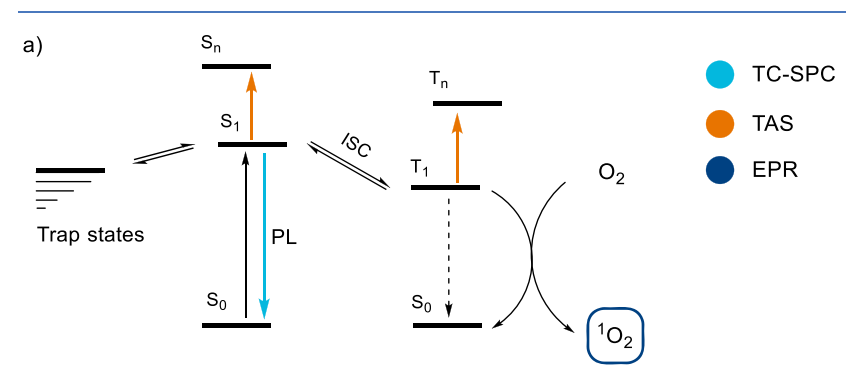

b)

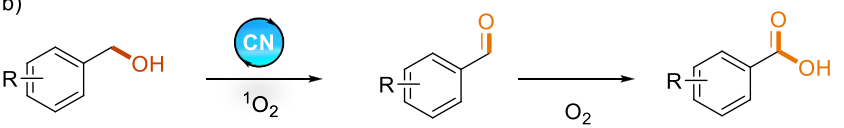

Figure 5. Mechanism of benzyl alcohol oxidation using thin $\mathrm{CN}$ films. (a) Proposed photophysical mechanism. (b) Photocatalytic mechanism of benzyl alcohol oxidation to benzaldehyde and subsequent thermal oxidation to benzoic acid.

Absorption of light by the $\mathrm{CN}$ thin film leads to the formation of the $\mathrm{CN}$ singlet excited states $\left(\mathrm{S}_{1}\right)$. Part of the singlet excited states undergoes ISC to form triplet excited states $\left(T_{1}\right)$ characterized by lifetime, which are long enough to interact with $\mathrm{O}_{2}$ via the EnT mechanism. Indeed, tr-PL data acquired for the $\mathrm{CN}$ thin films in oxygenated and degassed $\mathrm{CH}_{3} \mathrm{CN}$ clearly indicate that singlet excited states are not quenched by oxygen $-S_{1}$ life time is ca. 0.6 ns regardless of the environment (Table S4). On the other hand, TAS data indicate that $\mathrm{O}_{2}$ does quench triplet excited states as its lifetime decreases from 238 to $158 \mathrm{~ns}$ (Table S5). Once formed, ${ }^{1} \mathrm{O}_{2}$ reacts with benzyl alcohol producing benzaldehyde and presumably $\mathrm{H}_{2} \mathrm{O}_{2}{ }^{68}$ Then, benzaldehyde is converted to benzoic acid either via the thermal or photocatalytic pathway (Figure $5 b$ ).

Hence, the developed technology not only makes the VisBWR a convenient choice to conduct a photocatalytic reaction but is also more efficient compared to the analogous dispersion of the photocatalyst in the liquid medium. 


\section{CONCLUSIONS}

In this work, we presented an innovative approach in visible photocatalysis exploiting $\mathrm{CN}$ thin films.

A common lab vial was coated employing CVD becoming a reusable reactor itself. We exploited the as-coated vials as photoreactors in the one-step oxidation of 10 alcohols, in which corresponding benzoic acids have been obtained with up to $87 \%$ yield in $24 \mathrm{~h}$. Based on EPR, time-resolved emission and absorption spectroscopy, oxidation of benzyl alcohols mediated by the $\mathrm{CN}$ thin films involves singlet oxygen, which is sensitized via EnT from triplet excited states. Furthermore, we applied this technology also to microfluidic systems by coating the two mirror parts of a microfluidic flow reactor. The latter was successfully tested in the oxidation of five different substituted benzyl alcohols, selectively leading to benzaldehydes with excellent yield with much shorter reaction time. We believe that the technology developed herein is a significant step forward for the improvement of photocatalysis, both at a research and industrial level, because it allows us to craft different photocatalytic tools by coating diverse objects with complex geometries, otherwise out of reach with other preparative methods. Indeed, the utilization of thin-film technology allows us to truly implement photocatalysis in automated labs, such as HTS, as no time-demanding postseparation steps of the heterogeneous photocatalysts are required, such as filtration or centrifugation. Furthermore, using $\mathrm{CN}$ thin films, we further overcome the typical problems occurring also in microfluidic flow reactors with heterogeneous photocatalysts, such as fouling and clogging of the reactor and/ or pumping system. Exploiting $\mathrm{CN}$ thin films would also allow us to make more complicated tandem or cascade reaction setups, by combining different flow reactors, or graft from the surface desired functional species in a desired position, such as small molecules, polymers, and eventually enzymes. Beyond that, the presented technology can be exploited not only for photocatalysis, but furthermore for electro- and thermocatalyses or any of their combination. Eventually, we believe that the presented technology will pave the way for new, safer, more sustainable and thereby exciting chemistry, combining the advantages of thin films and (photo-)catalysis, whereby limits are dictated by scientist imagination.

\section{ASSOCIATED CONTENT}

\section{SI Supporting Information}

The Supporting Information is available free of charge at https://pubs.acs.org/doi/10.1021/acscatal.1c02909.

Methods; material characterization; photocatalytic and photophysical studies; products characterization (PDF)

\section{AUTHOR INFORMATION}

\section{Corresponding Authors}

Aleksandr Savateev - Max-Planck Institute of Colloids and Interfaces, Department of Colloid Chemistry, Potsdam 14476, Germany; 이잉.org/0000-0002-5760-6033; Email: oleksandr.savatieiev@mpikg.mpg.de

Paolo Giusto - Max-Planck Institute of Colloids and Interfaces, Department of Colloid Chemistry, Potsdam 14476, Germany; 이잉.org/0000-0003-4181-6500; Email: paolo.giusto@mpikg.mpg.de

\section{Authors}

Stefano Mazzanti - Max-Planck Institute of Colloids and Interfaces, Department of Colloid Chemistry, Potsdam 14476, Germany; 이이이.org/0000-0002-8704-051X

Giovanni Manfredi - Center for Nano Science and Technology, Istituto Italiano di Tecnologia, Milan 20133, Italy; 1 orcid.org/0000-0003-0682-0731

Alex J. Barker - Center for Nano Science and Technology, Istituto Italiano di Tecnologia, Milan 20133, Italy; (i) orcid.org/0000-0002-5614-4113

Markus Antonietti - Max-Planck Institute of Colloids and Interfaces, Department of Colloid Chemistry, Potsdam 14476, Germany; 이이.org/0000-0002-8395-7558

Complete contact information is available at:

https://pubs.acs.org/10.1021/acscatal.1c02909

\section{Funding}

Open access funded by Max Planck Society.

\section{Notes}

The authors declare no competing financial interest.

\section{ACKNOWLEDGMENTS}

We gratefully acknowledge the Max-Planck Society for generous financial support. The Deutsche Forschungsgemeinschaft is gratefully acknowledged for providing financial support for this project (DFG-An 156 13-1). P.G. wants to acknowledge Dr. Baris Kumru, Dr. Majd Al-Naji, Francesco Brandi, and Regina Rothe for the help and fruitful discussion during the development of this project. Furthermore, we acknowledge Rehinild Duennebacke, Irina Berndt for AFM measurements, Bortoluya Bodomoori for TEM and EELS measurements, Heike Runge for SEM and EDX measurements, and Enrico Lepre and Katharina ten Brummelhuis for nitrogen adsorption/desorption measurements.

\section{REFERENCES}

(1) Hoffmann, N. Photochemical reactions as key steps in organic synthesis. Chem. Rev. 2008, 108, 1052-1103.

(2) Balzani, V.; Bergamini, G.; Ceroni, P. Light: a very peculiar reactant and product. Angew. Chem., Int. Ed. 2015, 54, 11320-11337.

(3) Scholes, G. D.; Mirkovic, T.; Turner, D. B.; Fassioli, F.; Buchleitner, A. Solar light harvesting by energy transfer: from ecology to coherence. Energy Environ. Sci. 2012, 5, 9374-9393.

(4) Cambié, D.; Bottecchia, C.; Straathof, N. J. W.; Hessel, V.; Noël, T. Applications of Continuous-Flow Photochemistry in Organic Synthesis, Material Science, and Water Treatment. Chem. Rev. 2016, 116, 10276-10341.

(5) Schultz, D.; Campeau, L.-C. Harder, better, faster. Nat. Chem. 2020, 12, 661-664.

(6) Pipal, R. W.; Stout, K. T.; Musacchio, P. Z.; Ren, S.; Graham, T. J. A.; Verhoog, S.; Gantert, L.; Lohith, T. G.; Schmitz, A.; Lee, H. S.; Hesk, D.; Hostetler, E. D.; Davies, I. W.; MacMillan, D. W. C. Metallaphotoredox aryl and alkyl radiomethylation for PET ligand discovery. Nature 2021, 589, 542-547.

(7) Dighe, S. U.; Luridiana, A.; Douglas, J. J.; Leonori, D.; Leonori, D. A photochemical dehydrogenative strategy for aniline synthesis. Nature 2020, 584, 75-81.

(8) Ruffoni, A.; Juliá, F.; Svejstrup, T. D.; McMillan, A. J.; Douglas, J. J.; Leonori, D. Practical and regioselective amination of arenes using alkyl amines. Nat. Chem. 2019, 11, 426-433.

(9) Liang, Y.; Zhang, X.; MacMillan, D. W. C. Decarboxylative sp3 C-N coupling via dual copper and photoredox catalysis. Nature 2018, $559,83-88$. 
(10) Johnston, C. P.; Smith, R. T.; Allmendinger, S.; MacMillan, D. W. C. Metallaphotoredox-catalysed sp3-sp3 cross-coupling of carboxylic acids with alkyl halides. Nature 2016, 536, 322-325.

(11) Markushyna, Y.; Smith, C. A.; Savateev, A. Organic Photocatalysis: Carbon Nitride Semiconductors vs. Molecular Catalysts. Eur. J. Org. Chem. 2020, 2020, 1294-1309.

(12) Rajeshwar, K.; Thomas, A.; Janáky, C. Photocatalytic Activity of Inorganic Semiconductor Surfaces: Myths, Hype, and Reality. J. Phys. Chem. Lett. 2015, 6, 139-147.

(13) Li, R.; Zhang, F.; Wang, D.; Yang, J.; Li, M.; Zhu, J.; Zhou, X.; Han, H.; Li, C. Spatial separation of photogenerated electrons and holes among $\{010\}$ and $\{110\}$ crystal facets of BiVO4. Nat. Commun. 2013, 4, 1432.

(14) Li, L.; Salvador, P. A.; Rohrer, G. S. Photocatalysts with internal electric fields. Nanoscale 2014, 6, 24-42.

(15) Mazzanti, S.; Savateev, A. Emerging Concepts in Carbon Nitride Organic Photocatalysis. ChemPlusChem 2020, 85, 24992517.

(16) Strieth-Kalthoff, F.; James, M. J.; Teders, M.; Pitzer, L.; Glorius, F. Energy transfer catalysis mediated by visible light: principles, applications, directions. Chem. Soc. Rev. 2018, 47, 7190-7202.

(17) Wang, X.; Maeda, K.; Thomas, A.; Takanabe, K.; Xin, G.; Carlsson, J. M.; Domen, K.; Antonietti, M. A metal-free polymeric photocatalyst for hydrogen production from water under visible light. Nat. Mater. 2009, 8, 76.

(18) Rosso, C.; Filippini, G.; Criado, A.; Melchionna, M.; Fornasiero, P.; Prato, M. Metal-Free Photocatalysis: Two-Dimensional Nanomaterial Connection toward Advanced Organic Synthesis. ACS Nano 2021, 15, 3621-3630.

(19) Savateev, A.; Ghosh, I.; König, B.; Antonietti, M. Photoredox Catalytic Organic Transformations using Heterogeneous Carbon Nitrides. Angew. Chem., Int. Ed. 2018, 57, 15936-15947.

(20) Savateev, A.; Antonietti, M. Ionic Carbon Nitrides in Solar Hydrogen Production and Organic Synthesis: Exciting Chemistry and Economic Advantages. ChemCatChem 2019, 11, 6166-6176.

(21) Savateev, A.; Tarakina, N. V.; Strauss, V.; Hussain, T.; Brummelhuis, K.; Sánchez Vadillo, J. M.; Markushyna, Y.; Mazzanti, S.; Tyutyunnik, A. P.; Walczak, R.; Oschatz, M.; Guldi, D. M.; Karton, A.; Antonietti, M. Potassium Poly(Heptazine Imide): Transition Metal-Free Solid-State Triplet Sensitizer in Cascade Energy Transfer and [3+2]-cycloadditions. Angew. Chem., Int. Ed. 2020, 59, 1506115068 .

(22) Wang, H.; Jiang, S.; Chen, S.; Zhang, X.; Shao, W.; Sun, X.; Zhao, Z.; Zhang, Q.; Luo, Y.; Xie, Y. Insights into the excitonic processes in polymeric photocatalysts. Chem. Sci. 2017, 8, 40874092.

(23) Rieth, A. J.; Qin, Y.; Martindale, B. C. M.; Nocera, D. G. LongLived Triplet Excited State in a Heterogeneous Modified Carbon Nitride Photocatalyst. J. Am. Chem. Soc. 2021, 143, 4646-4652.

(24) Das, S.; Murugesan, K.; Villegas Rodríguez, G. J.; Kaur, J.; Barham, J. P.; Savateev, A.; Antonietti, M.; König, B. Photocatalytic (Het)arylation of C(sp3)-H Bonds with Carbon Nitride. ACS Catal. 2021, 11, 1593-1603.

(25) Ola, O.; Maroto-Valer, M. M. Review of material design and reactor engineering on $\mathrm{TiO} 2$ photocatalysis for $\mathrm{CO} 2$ reduction. J. Photochem. Photobiol., C 2015, 24, 16-42.

(26) Shwetharani, R.; Chandan, H. R.; Sakar, M.; Balakrishna, G. R.; Reddy, K. R.; Raghu, A. V. Photocatalytic semiconductor thin films for hydrogen production and environmental applications. Int. J. Hydrogen Energy 2020, 45, 18289-18308.

(27) Yang, C.; Li, R.; Zhang, K. A. I.; Lin, W.; Landfester, K.; Wang, $\mathrm{X}$. Heterogeneous photoredox flow chemistry for the scalable organosynthesis of fine chemicals. Nat. Commun. 2020, 11, 1239.

(28) Pierson, H. O. Introduction and General Considerations. In Handbook of Chemical Vapor Deposition (CVD), 2nd ed.; Pierson, H. O., Ed.; William Andrew Publishing: Norwich, NY, 1999; pp 25-35.

(29) Bian, J.; Li, J.; Kalytchuk, S.; Wang, Y.; Li, Q.; Lau, T. C.; Niehaus, T. A.; Rogach, A. L.; Zhang, R.-Q. Efficient Emission Facilitated by Multiple Energy Level Transitions in Uniform Graphitic
Carbon Nitride Films Deposited by Thermal Vapor Condensation. ChemPhysChem 2015, 16, 954-959.

(30) Arazoe, H.; Miyajima, D.; Akaike, K.; Araoka, F.; Sato, E.; Hikima, T.; Kawamoto, M.; Aida, T. An autonomous actuator driven by fluctuations in ambient humidity. Nat. Mater. 2016, 15, 10841089.

(31) Peng, G.; Albero, J.; Garcia, H.; Shalom, M. A Water-Splitting Carbon Nitride Photoelectrochemical Cell with Efficient Charge Separation and Remarkably Low Onset Potential. Angew. Chem., Int. Ed. 2018, 57, 15807-15811.

(32) Xiong, W.; Huang, F.; Zhang, R.-Q. Recent developments in carbon nitride based films for photoelectrochemical water splitting. Sustainable Energy Fuels 2020, 4, 485-503.

(33) Zhang, J.; Zou, Y.; Eickelmann, S.; Njel, C.; Heil, T.; Ronneberger, S.; Strauss, V.; Seeberger, P. H.; Savateev, A.; Loeffler, F. F. Laser-driven growth of structurally defined transition metal oxide nanocrystals on carbon nitride photoelectrodes in milliseconds. Nat. Commun. 2021, 12, 3224.

(34) Qin, J.; Barrio, J.; Peng, G.; Tzadikov, J.; Abisdris, L.; Volokh, M.; Shalom, M. Direct growth of uniform carbon nitride layers with extended optical absorption towards efficient water-splitting photoanodes. Nat. Commun. 2020, 11, 4701.

(35) Giusto, P.; Cruz, D.; Heil, T.; Arazoe, H.; Lova, P.; Aida, T.; Comoretto, D.; Patrini, M.; Antonietti, M. Shine Bright Like a Diamond: New Light on an Old Polymeric Semiconductor. Adv. Mater. 2020, 32, 1908140.

(36) Giusto, P.; Kumru, B.; Zhang, J.; Rothe, R.; Antonietti, M. Let a Hundred Polymers Bloom: Tunable Wetting of Photografted Polymer-Carbon Nitride Surfaces. Chem. Mater. 2020, 32, 72847291.

(37) Wang, X.; Maeda, K.; Thomas, A.; Takanabe, K.; Xin, G.; Carlsson, J. M.; Domen, K.; Antonietti, M. A metal-free polymeric photocatalyst for hydrogen production from water under visible light. Nat. Mater. 2009, 8, 76-80.

(38) Mazzanti, S.; Cao, S.; ten Brummelhuis, K.; Völkel, A.; Khamrai, J.; Sharapa, D. I.; Youk, S.; Heil, T.; Tarakina, N. V.; Strauss, V.; Ghosh, I.; König, B.; Oschatz, M.; Antonietti, M.; Savateev, A. Allorganic Z-scheme photoreduction of $\mathrm{CO} 2$ with water as the donor of electrons and protons. Appl. Catal., B 2021, 285, 119773.

(39) Ghosh, I.; Khamrai, J.; Savateev, A.; Shlapakov, N.; Antonietti, M.; König, B. Organic semiconductor photocatalyst can bifunctionalize arenes and heteroarenes. Science 2019, 365, 360.

(40) Savateev, A.; Antonietti, M. Heterogeneous Organocatalysis for Photoredox Chemistry. ACS Catal. 2018, 8, 9790-9808.

(41) Khamrai, J.; Ghosh, I.; Savateev, A.; Antonietti, M.; König, B. Photo-Ni-Dual-Catalytic C(sp2)-C(sp3) Cross-Coupling Reactions with Mesoporous Graphitic Carbon Nitride as a Heterogeneous Organic Semiconductor Photocatalyst. ACS Catal. 2020, 10, 35263532.

(42) Mazzanti, S.; Kurpil, B.; Pieber, B.; Antonietti, M.; Savateev, A. Dichloromethylation of enones by carbon nitride photocatalysis. Nat. Commun. 2020, 11, 1387.

(43) Filippini, G.; Longobardo, F.; Forster, L.; Criado, A.; Di Carmine, G.; Nasi, L.; D’Agostino, C.; Melchionna, M.; Fornasiero, P.; Prato, M. Light-driven, heterogeneous organocatalysts for C-C bond formation toward valuable perfluoroalkylated intermediates. Sci. $A d v .2020,6$, No. eabc9923.

(44) Vijeta, A.; Casadevall, C.; Roy, S.; Reisner, E. Visible-Light Promoted C-O Bond Formation with an Integrated Carbon NitrideNickel Heterogeneous Photocatalyst. Angew. Chem., Int. Ed. 2021, 60, 8494-8499.

(45) Kumru, B.; Antonietti, M. Colloidal properties of the metal-free semiconductor graphitic carbon nitride. Adv. Colloid Interface Sci. 2020, 283, 102229.

(46) Cruz, D.; Garcia Cerrillo, J.; Kumru, B.; Li, N.; Dario Perea, J.; Schmidt, B. V. K. J.; Lauermann, I.; Brabec, C. J.; Antonietti, M. Influence of Thiazole-Modified Carbon Nitride Nanosheets with Feasible Electronic Properties on Inverted Perovskite Solar Cells. J. Am. Chem. Soc. 2019, 141, 12322-12328. 
(47) Jia, C.; Yang, L.; Zhang, Y.; Zhang, X.; Xiao, K.; Xu, J.; Liu, J. Graphitic Carbon Nitride Films: Emerging Paradigm for Versatile Applications. ACS Appl. Mater. Interfaces 2020, 12, 53571-53591.

(48) Liu, J.; Wang, H.; Antonietti, M. Graphitic carbon nitride "reloaded": emerging applications beyond (photo) catalysis. Chem. Soc. Rev. 2016, 45, 2308-2326.

(49) Xu, J.; Shalom, M.; Piersimoni, F.; Antonietti, M.; Neher, D.; Brenner, T. J. K. Color-Tunable Photoluminescence and NIR Electroluminescence in Carbon Nitride Thin Films and LightEmitting Diodes. Adv. Opt. Mater. 2015, 3, 913-917.

(50) Despite CN film prepared by CVD has been characterized earlier, we consider necessary to provide full characterization data as properties of the films may depend on the employed substrate.

(51) Signal of silicon (Figure 2d) and oxygen (Figure S4f) originate from the substrate and greatly enhanced at the area, where the film has been removed.

(52) Mane, G. P.; Dhawale, D. S.; Anand, C.; Ariga, K.; Ji, Q.; Wahab, M. A.; Mori, T.; Vinu, A. Selective sensing performance of mesoporous carbon nitride with a highly ordered porous structure prepared from 3-amino-1,2,4-triazine. J. Mater. Chem. A 2013, 1, 2913-2920.

(53) Sun, Y.; Yuan, J. Electron energy loss spectroscopy of coreelectron excitation in anisotropic systems: Magic angle, magic orientation, and dichroism. Phys. Rev. B 2005, 71, 125109.

(54) Jouffrey, B.; Schattschneider, P.; Hébert, C. The magic angle: a solved mystery. Ultramicroscopy 2004, 102, 61-66.

(55) Dvoranová, D.; Barbieriková, Z.; Brezová, V. Radical intermediates in photoinduced reactions on $\mathrm{TiO} 2$ (an EPR spin trapping study). Molecules 2014, 19, 17279-17304.

(56) Sankar, M.; Nowicka, E.; Carter, E.; Murphy, D. M.; Knight, D. W.; Bethell, D.; Hutchings, G. J. The benzaldehyde oxidation paradox explained by the interception of peroxy radical by benzyl alcohol. Nat. Commun. 2014, 5, 3332.

(57) Bajada, M. A.; Vijeta, A.; Savateev, A.; Zhang, G.; Howe, D.; Reisner, E. Visible-Light Flow Reactor Packed with Porous Carbon Nitride for Aerobic Substrate Oxidations. ACS Appl. Mater. Interfaces 2020, 12, 8176-8182.

(58) Burger, B.; Maffettone, P. M.; Gusev, V. V.; Aitchison, C. M.; Bai, Y.; Wang, X.; Li, X.; Alston, B. M.; Li, B.; Clowes, R.; Rankin, N.; Harris, B.; Sprick, R. S.; Cooper, A. I. A mobile robotic chemist. Nature 2020, 583, 237-241.

(59) Savateev, A.; Pronkin, S.; Epping, J. D.; Willinger, M. G.; Wolff, C.; Neher, D.; Antonietti, M.; Dontsova, D. Potassium Poly(heptazine imides) from Aminotetrazoles: Shifting Band Gaps of Carbon Nitridelike Materials for More Efficient Solar Hydrogen and Oxygen Evolution. ChemCatChem 2017, 9, 167-174.

(60) Gérardy, R.; Emmanuel, N.; Toupy, T.; Kassin, V. E.; Tshibalonza, N. N.; Schmitz, M.; Monbaliu, J. C. M. Continuous flow organic chemistry: successes and pitfalls at the interface with current societal challenges. Eur. J. Org. Chem. 2018, 2018, 23012351.

(61) Godin, R.; Wang, Y.; Zwijnenburg, M. A.; Tang, J.; Durrant, J. R. Time-Resolved Spectroscopic Investigation of Charge Trapping in Carbon Nitrides Photocatalysts for Hydrogen Generation. J. Am. Chem. Soc. 2017, 139, 5216-5224.

(62) Talapaneni, S. N.; Singh, G.; Kim, I. Y.; AlBahily, K.; AlMuhtaseb, A. a. H.; Karakoti, A. S.; Tavakkoli, E.; Vinu, A. Nanostructured Carbon Nitrides for CO 2 Capture and Conversion. Adv. Mater. 2020, 32, 1904635.

(63) Phillips, J. C. Microscopic aspects of Stretched Exponential Relaxation (SER) in homogeneous molecular and network glasses and polymers. J. Non-Cryst. Solids 2011, 357, 3853-3865.

(64) Changes in absorbance maximum from one sample to the other can be attributed to changes in the grating position whose efficiency greatly drops near the edges of the studied wavelength intervals.

(65) Mikhnenko, O. V.; Cordella, F.; Sieval, A. B.; Hummelen, J. C.; Blom, P. W. M.; Loi, M. A. Temperature Dependence of Exciton Diffusion in Conjugated Polymers. J. Phys. Chem. B 2008, 112, 11601-11604.
(66) Jia, W.; Wang, Q.; Shi, H.; An, Z.; Huang, W. Manipulating the Ultralong Organic Phosphorescence of Small Molecular Crystals. Chem.-Eur. J. 2020, 26, 4437-4448.

(67) Marschall, R. 50 Years of Materials Research for Photocatalytic Water Splitting. Eur. J. Inorg. Chem. 2021, 2021, 2435-2441.

(68) Arcas, R.; Peris, E.; Mas-Marzá, E.; Fabregat-Santiago, F. Revealing the contribution of singlet oxygen in the photoelectrochemical oxidation of benzyl alcohol. Sustainable Energy Fuels 2021, 5, 956-962. 